\title{
Regional Cerebral Gray Matter Atrophy Is Associated With Cognitive Impairment in Hemodialysis Patients: a Cross-sectional and Longitudinal Voxel-based Morphological Mri Study
}

\section{Huiying Wang}

The School of Medicine $₫$ Nankai University

\section{Lixiang Huang}

Department of Radiology, The Second Hospital of Tianjin Medical University

\section{Gemuer Wu}

The School of Medicine, Nankai University

Jinping Li

Tianjin First Central Hospital, School of Medicine, Nankai University

Lei Liu

Hebei Petrochina Central Hospital

\section{Tong Zhang}

First Central Clinical College, Medical Uniersity of Tianjin

\section{Jinxia Zhu}

Siemens Healthcare Pte Ltd

\section{Xianchang Zhang}

Siemens Healthcare Pte Ltd

\section{Wen Shen}

Tianjin First Central Hospital, School of Medicine, Nankai University

\section{Chao Chai}

Tianjin First Cental Hospital, School of Medicine, Nankai University

Shuang Xia ( $\square$ xiashuang77@163.com)

Tianjin First Centerl Hospital, School of Medicine, Nankai University https://orcid.org/0000-0003-1626-6383

\section{Research Article}

Keywords: Kidney Failure, Chronic, Renal Dialysis, Magnetic Resonance Imaging, Gray matter, Cognitive Dysfunction

Posted Date: May 5th, 2021

DOI: https://doi.org/10.21203/rs.3.rs-480949/v1

License: (a) (1) This work is licensed under a Creative Commons Attribution 4.0 International License. Read Full License 
Version of Record: A version of this preprint was published at Brain Imaging and Behavior on January 7th, 2022. See the published version at https://doi.org/10.1007/s11682-021-00602-9. 


\section{Abstract}

To explore changes of cerebral gray matter volumes (GMV) in hemodialysis patients, the clinical risk factors for GMV changes, and the relationship between GMV changes and neuropsychological tests results. Eighty-eight hemodialysis patients and seventy-six healthy controls (HCs) were recruited in this study. Fifty patients underwent follow-up magnetic resonance, blood biochemical examinations, and neuropsychological test (1.75 \pm 0.55 years). Changes in GMV between patients and HCs, longitudinal GMV changes in patients were explored. The clinical risk factors for the longitudinal GMV changes, the correlations between longitudinal GMV changes and neuropsychological tests changes in hemodialysis patients were analyzed. Compared to HCs, the hemodialysis patients had diffusely decreased in GMV (with age, sex, and total intracranial volume [TIV] as covariates, $P<0.001$, voxel-wise threshold false discovery rate [FDR] corrected). Compared with patients at baseline, regional decreased GMV were found in patients at follow-up (with age, and TIV as covariates, $P<0.05$, voxel-wise threshold FDR corrected). Increased serum urea, parathyroid hormone levels, and hemodialysis duration were independent risk factors for decreased GMV in hemodialysis patients (all $P<0.05$, FDR corrected). Hemodialysis patients had lower MMSE $(27[26,29])$ and MoCA $(22[19.48,24.04])$ scores than $\mathrm{HCs}(30[29,30]$ and $28[26.89,29])($ all $P<0.05)$. The MMSE scores of hemodialysis patients at follow-up $(26[25,28.5])$ were lower than those of patients at baseline $(28[25,29.5])(P=0.02)$. The decreased left caudate volumes were positively correlated with reduced MMSE scores in hemodialysis patients $\left(r_{s}=0.437, P=0.033\right)$. Hemodialysis patients had diffused $\mathrm{GM}$ atrophy over time, which was related to cognitive impairment.

\section{Introduction}

Kidney failure is defined as severe and irreversible kidney function impairment with an estimated glomerular filtration rate of $<15 \mathrm{~mL} / \mathrm{min} / 1.73 \mathrm{~m}^{2}$ (GRF category G5) ${ }^{1}$. Although hemodialysis has been an effectively and widely used treatment for kidney failure, it can often cause central nervous system complications, including cerebrovascular diseases ${ }^{2}$, leukoencephalopathy ${ }^{3}$, and cognitive impairment ${ }^{4}$. These complications may lead to poor clinical outcomes, such as hospitalization and disability ${ }^{5,6}$. Therefore, early diagnosis of central nervous system complications is vital to prevent the progression of these complications and provide the optimal treatment.

Recently MR techniques have played an important role in the early diagnosis of central nervous systems complications in hemodialysis patients. These techniques included resting-state functional MR imaging ${ }^{7}$, diffusion tensor imaging $(\mathrm{DTI})^{8}$, arterial spin labeling $(\mathrm{ASL})^{9}$, magnetic resonance spectroscopy $(\mathrm{MRS})^{10}$, and susceptibility weighted imaging $(\mathrm{SWI})^{11}$. The application of these techniques has shown that there are abnormal brain changes in hemodialysis patients, such as impairments of functional connectivity ${ }^{12}$, disruptions of white matter integrity ${ }^{8}$, cerebral blood flow changes ${ }^{9}$, abnormal brain metabolism ${ }^{10}$, and cerebral microbleeds ${ }^{11}$. However, it has been reported that morphological changes can more directly reflect abnormal brain changes in hemodialysis patients ${ }^{13}$. To support this finding, brain atrophy has been assessed using predefined visual standards in hemodialysis patients ${ }^{14}$. However, this method was insensitive to microstructure changes and heavily depended on the experience levels of observers. Voxel-based morphometry (VBM) has been widely used to detect microstructure changes in patients with kidney failure ${ }^{15}$, hemodialysis ${ }^{16,17}$, and chronic kidney disease (CKD) ${ }^{18}$. All of these studies found gray matter (GM) and white matter (WM) atrophy. However, most studies were cross-sectional, which was affected by the differences among the individuals and further caused the inaccuracies in the evaluation of cerebral volumes. To date, only Tsuruya et al. ${ }^{18}$ explored longitudinal brain volumes changes in CKD and peritoneal 
dialysis patients and found that peritoneal dialysis patients had severe GM atrophy compared to CKD patients. It has also been reported that hemodialysis and peritoneal dialysis were different dialysis modalities and have different adverse effect on the cerebral structures ${ }^{19}$. However, few studies explored the effect of only regular hemodialysis therapy on the cerebral structures.

All these above VBM analyses were performed with the VBM8 toolbox implemented in Statistical Parametric Mapping 8 (SPM8) software. Recently, a new VBM toolbox called Computational Anatomy Toolbox (CAT) was introduced by a Structural Brain Mapping group (http://www.neuro.uni-jena.de/cat/). Compared to the previous VBM8 toolbox, the CAT12 toolbox is easier to operate because of increased automation, and more accurate and robust results ${ }^{20}$. It has been reported that CAT12 has improved the normalization and segmentation during the VBM analysis ${ }^{20}$. In recent years, the CAT12 toolbox had been widely used in brain volume analyses of diseases including Parkinson's disease ${ }^{21}$, Alzheimer's disease ${ }^{22}$, and depressive disorder ${ }^{23}$. To date, there has been no studies to explore the longitudinal brain volumes changes in hemodialysis patients using the CAT12 toolbox.

We hypothesized that the process of hemodialysis may cause the aggravation of brain atrophy, which correlated with cognitive impairment. The purpose of this study was to investigate: 1) the brain volume changes in hemodialysis patients by cross-sectional and longitudinal MRI examinations using the CAT12 toolbox; 2) the clinical risk factors for the gray matter volume (GMV) changes; and 3) the correlations between brain volumes changes and cognitive function changes.

\section{Methods}

\section{Subjects}

This study was approved by the Medical Research Ethics Committee of our hospital (2015N002KY) and all the subjects offered their written informed consent form. A total of 97 hemodialysis patients were recruited from the Hemodialysis Center of the hospital from November 2015 to July 2019. The inclusion criteria were: 1) all the subjects were 18 years or older and right-handed; 2) all the patients completed the baseline and follow-up examinations to acquire all available MRI data; 3 ) the dialysis duration of patients was more than 3 months without history of peritoneal dialysis or kidney transplantation. The exclusion criteria included: 1) inability to complete the MRI examinations; 2) the subjects had other diseases affecting brain volumes including chronic infarction, hematoma, trauma, and some neurodegenerative diseases including Alzheimer's disease, Parkinson's disease, etc.; 3) congenital brain abnormalities were found; 4) the image quality was too poor to perform VBM analysis. Based on the inclusion and exclusion criteria, 88 patients were enrolled (Supplementary Fig. 1). Each patient underwent the laboratory examination, neuropsychological and brain MRI assessment at baseline. The majority of them (50 of 88 ) received second examinations at follow-up. Finally, only 33 patients who met the inclusion and exclusion criteria were enrolled as follow-up group (1.75 \pm 0.55 years) (Supplementary Fig. 1). All patients received regular hemodialysis therapy for four hours each time, three times a week. Seventy-six age-, sex-, and education-matched HCs were recruited from local communities in this city. HCs did not have the follow-up examinations.

\section{Laboratory examinations and neuropsychological tests}

To investigate the clinical risk factors for the brain volume changes in hemodialysis patients, blood biochemical examinations were performed within a week before MR scanning. The blood biochemical indices included 
hemoglobin, serum urea, serum creatinine, serum phosphorus, calcium, and parathyroid hormone according to the previous studies and relative literatures ${ }^{24,25}$. No blood biochemical tests were performed on HCs.

In order to assess cognitive function of all subjects, Mini-mental State Examination (MMSE) and Montreal Cognitive Assessment (MoCA) tests were performed by a trained neuroradiologist (T.Z., who was trained by a 20 years' experienced neuropsychologist and blinded to the clinical information) in a quite environment the day before MR scanning. The MMSE and MoCA tests were used to evaluate the cognitive function including orientation, registration, attention, calculation, recall, language and praxis. They had been widely applied in the cognitive evaluation of kidney failure and hemodialysis patients ${ }^{26}$.

\section{Imaging data acquisition and analysis}

All MRI data were acquired on a 3 T MR imager (MAGNETOM Trio a Tim System, Siemens Healthcare) using an 8channel phased-array head coil. All subjects were required to keep their head immobile during the MR examination. First, conventional MR sequences, including $\mathrm{T}_{2} \mathrm{WI}$ and $\mathrm{T}_{2}$ fluid attenuated inversion recovery (FLAIR) were applied to exclude the congenital cerebral abnormalities, occupying-space diseases, and demyelinating diseases. Then, high resolution $3 \mathrm{D}$ sagittal $\mathrm{T}_{1}$-weighted images were obtained using magnetization-prepared rapid gradient-echo (MP-RAGE) sequence. The parameters of each sequences were presented in the Supplemental Materials.

The CAT12 Toolbox was used to perform VBM analyses within SPM12 (Statistical Parametric Mapping, Institute of Neurology, London, UK) running on MATLAB (R2010b; MathWorks). For the patient's longitudinal data, we used longitudinal data segmentation to preprocess those images. The postprocessing steps of VBM analysis included the following steps: (1) All $\mathrm{T}_{1}$ structure images were normalized and were bias-field corrected. (2) All images were aligned to Montreal Neurological Institute (MNI) standard space. (3) These images were segmented into white matter, GM, and cerebrospinal fluid. (4) After imaging segmentation, images of all subjects were checked to ensure to be further used for subsequent analysis. (5) A statistical analysis module was applied to estimate the total intracranial volume (TIV) of each subject. Finally, all data were smoothed on SPM12 with a kernel of $8 \mathrm{~mm}$ full width at half maximum (FWHM) ${ }^{27}$. GMV was divided by TIV to acquire the normalized GMV in order to remove the effect of cerebral size variability.

\section{Statistical Analyses}

Statistical analyses were conducted using SPSS software (version 17.0, SPSS Inc.) and SPM12 package running on MATLAB (R2010b; MathWorks). First, the normal distribution of data was analyzed using the KolmogorovSmirnov test. The differences in age and education levels between hemodialysis patients and HCs were explored by Mann-Whitney U test. The differences in sex between hemodialysis patients and HCs were explored by Chisquare test. Two independent sample t test was employed to explore the differences in normalized GMV between patients and HCs at baseline. Paired-sample t test was conducted to evaluated the differences in normalized GMV between patients at baseline and follow-up. The differences in MMSE and MoCA scores between patients and HCs were investigated using the Mann-Whitney $U$ test. Because the majority of patients refused to undergo second MoCA tests (28/33 at follow-up group) due to the long time of neuropsychological tests, the differences of MoCA scores between baseline and follow-up group were not analyzed in this study. The differences in MMSE scores between patients at baseline and follow-up were investigated using the Wilcoxon signed-rank test.

The clinical risk factors for brain volume changes in hemodialysis patients were investigated by stepwise multiple regression analysis. The correlations between brain volumes changes and MMSE scores changes in patients were 
investigated using Spearman's correlation analysis. An FDR corrected $P<0.05$ for stepwise multiple regression analysis was considered as significant.

Statistical analyse of structural MRI data were carried out using SPM12. The differences in GMV between patients at baseline and HCs were explored using two independent sample t test (with age, sex, and TIV as covariates, voxelwise threshold of $P<0.001$, false discovery rate [FDR] corrected). The differences in GMV between baseline and follow-up examination in patients were investigated by paired-sample t test (with age and TIV as covariates, voxelwise threshold of $P<0.05$, FDR corrected).

\section{Results}

\section{Patient Characteristics}

The demographics and neuropsychological tests of the cross-sectional study were presented in Table 1 . There were no significant differences in age, sex, and education levels between patients and HCs $(P=0.627 ; 0.162 ; 0.875)$. The mean follow-up interval time was $1.75 \pm 0.55$ years. The MMSE scores of patients $(27[26,29])$ were significantly lower than $\mathrm{HCs}(30[29,30])(P<0.001)$. The MoCA scores of patients $(22[19.48,24.04])$ were significantly lower than HCs $(28[26.89,29])(P<0.001)$. The MMSE scores of hemodialysis patients at follow-up $(26[25,28.5])$ were significantly lower than those of patients at baseline $(28[25,29.5])(P=0.02)$. The characteristics,

neuropsychological tests, and laboratory examinations of the longitudinal study were shown in Table 2. Compared to patients at baseline, patients at follow-up had significantly higher parathyroid hormone levels $(P=0.026)$.

Table 1

Demographics and neuropsychological tests in the cross-sectional study

\begin{tabular}{|llll|}
\hline & HCs & patients & $P$ \\
\hline Age(years) & $46.5(35,53.75)$ & $47.5(33.25,57)$ & 0.627 \\
\hline Sex (M/F) & $34 / 42$ & $49 / 39$ & 0.162 \\
\hline Education level & $14(11,15)$ & $14(11,15)$ & $<.875$ \\
\hline MMSE & $30(29,30)$ & $27(26,29)$ & $<0.001^{\text {a }}$ \\
\hline MoCA & $28(26.89,29)$ & $22(19.48,24.04)$ & $<0.001^{\text {a }}$ \\
\hline Normalized GMV(\%) & $35.47 \pm 1.50$ & $34.14 \pm 1.90$ & 0.187 \\
\hline GMV & $520.30 \pm 64.87$ & $507.28 \pm 61.04$ & 0.381 \\
\hline TIV & $1464.40 \pm 148.05$ & $1483.88 \pm 135.94$ & \\
\hline $\begin{array}{l}\text { Data conforming to normal distribution are expressed by mean } \pm \text { standard deviation; data consistent with } \\
\text { skewed distributions are represented by medians (percentiles25th, percentiles75th). }\end{array}$ \\
\hline $\begin{array}{l}\text { HCs = Healthy Controls, MMSE = Mini-mental State Examination, MoCA = Montreal Cognitive Assessment, GMV } \\
\text { = Gray Matter Volume, TIV = Total Intracranial Volume }\end{array}$ \\
\hline \begin{tabular}{l} 
a Mann-Whitney U test \\
\hline b
\end{tabular}
\end{tabular}


Table 2

Characteristics, neuropsychological tests, and laboratory examinations in final included patients from the longitudinal study

\begin{tabular}{|c|c|c|c|}
\hline & Baseline & Follow up & $P$ \\
\hline MMSE & $28(25,29.5)$ & $26(25,28.5)$ & $0.02^{a}$ \\
\hline Normalized GMV(\%) & $33.92 \pm 2.23$ & $33.29 \pm 2.4$ & $0.001^{b}$ \\
\hline Urea & $27.71 \pm 9.10$ & $24.38 \pm 8.58$ & 0.063 \\
\hline Creatinine & $953.4 \pm 309.08$ & $815.04 \pm 417.94$ & 0.087 \\
\hline Serum phosphorus & $1.97 \pm 0.54$ & $1.80 \pm 0.65$ & 0.153 \\
\hline Parathyroid hormone & $130.2(64.46,226.3)$ & $213.1(116.5,408)$ & $0.026^{a}$ \\
\hline Hemoglobin & $113.56 \pm 3.16$ & $115.94 \pm 2.96$ & 0.53 \\
\hline \multicolumn{4}{|c|}{$\begin{array}{l}\text { Data conforming to normal distribution are expressed by mean } \pm \text { standard deviation; data consistent with } \\
\text { skewed distributions are represented by medians (percentiles } 25 \text { th, percentiles } 75 \text { th). }\end{array}$} \\
\hline \multicolumn{4}{|c|}{ MMSE = Mini-mental State Examination, GMV = Gray Matter Volume } \\
\hline \multicolumn{4}{|c|}{ a Wilcoxon signed ranks test } \\
\hline b Paired samples test & & & \\
\hline
\end{tabular}

\section{Brain volume changes between patients at baseline and $\mathrm{HCs}$}

The normalized GMV (\%) of patients (34.14 \pm 1.9$)$ were significantly lower than that of HCs $(35.47 \pm 1.5)(P \otimes 0.001)$ (Supplementary Fig. 2). Compared to HCs, patients showed diffusely decreased GMV in the bilateral frontal lobes, temporal lobes, occipital lobe, parietal lobe, limbic system, caudate, putamen, and globus pallidus (FDR corrected with age, sex, and TIV as covariates; voxel-wise threshold of $P<0.001$ ) (Fig. 1; Supplementary Table 1).

\section{Brain volume changes between baseline and follow-up}

The normalized GMV (\%) of patients at baseline (33.92 \pm 2.23$)$ were significantly lower than that of patients at follow-up (33.29 \pm 2.4$)(P=0.001)$ (Supplementary Fig. 2). Compared with patients at baseline, the patients at follow-up showed significantly decreased GMV in the bilateral frontal lobes, temporal lobes, insula, Rolandic operculum, caudate, right supplementary motor area, right precentral gyrus, right supramarginal gyrus, and right cingulate gyrus (with age and TIV as covariates, voxel-wise threshold of $P<0.05$, FDR corrected) (Fig. 2;

Supplementary Table 1).

\section{Clinical risk factors for brain volume changes in hemodialysis patients}

The independent risk factors for regional GMV changes included long hemodialysis duration, increased serum urea, and increased parathyroid hormone (Table 3). The Variance Inflation Factor (VIF) ranged from 1.000 to 1.008, indicating that there was no multicollinearity among the independent variables ${ }^{26}$. 
Table 3

Independent risk factors for brain volume changes in hemodialysis patients using stepwise multiple regression analysis

\begin{tabular}{|c|c|c|c|c|c|c|}
\hline $\begin{array}{l}\text { Regional brain volume } \\
\text { changes (AAL) }\end{array}$ & $\begin{array}{l}\text { Blood } \\
\text { biochemical } \\
\text { factors }\end{array}$ & $\begin{array}{l}\text { Standardized } \\
\text { coefficients }\end{array}$ & $\begin{array}{l}95 \% \\
\text { Confidence } \\
\text { interval }\end{array}$ & $\begin{array}{l}\text { Partial } \\
\text { correlation }\end{array}$ & $\begin{array}{l}\text { Collinearity } \\
\text { statistics } \\
\text { VIF }\end{array}$ & $P_{F D R}$ \\
\hline$\triangle$ Insula_L & Urea & 0.375 & $\begin{array}{l}(0.000 \\
0.001)\end{array}$ & 0.375 & 1.000 & 0.046 \\
\hline$\triangle$ Rolandic_Oper_L & Urea & 0.453 & $\begin{array}{l}(0.000, \\
0.002)\end{array}$ & 0.453 & 1.000 & 0.030 \\
\hline \multirow[t]{2}{*}{$\triangle$ Frontal_Sup_Medial_L } & Urea & 0.327 & $\begin{array}{l}(0.000, \\
0.000)\end{array}$ & 0.363 & 1.008 & 0.046 \\
\hline & $\begin{array}{l}\text { parathyroid } \\
\text { hormone }\end{array}$ & 0.410 & $(0.000,0.001)$ & 0.438 & 1.008 & 0.030 \\
\hline$\triangle$ Supp_Motor_Area_R & Urea & 0.384 & $\begin{array}{l}(0.000 \\
0.001)\end{array}$ & 0.348 & 1.000 & 0.045 \\
\hline \multirow[t]{2}{*}{$\triangle$ Frontal_Sup_Medial_R } & $\begin{array}{l}\text { parathyroid } \\
\text { hormone }\end{array}$ & 0.483 & $\begin{array}{l}(0.000 \\
0.000)\end{array}$ & 0.512 & 1.008 & 0.020 \\
\hline & Urea & 0.343 & $\begin{array}{l}(0.000 \\
0.001)\end{array}$ & 0.398 & 1.008 & 0.045 \\
\hline$\triangle$ Cingulum_Mid_R & $\begin{array}{l}\text { parathyroid } \\
\text { hormone }\end{array}$ & 0.445 & $(0.000,0.000)$ & 0.445 & 1.000 & 0.030 \\
\hline$\triangle$ Temporal_Sup_R & duration & 0.364 & $\begin{array}{l}(0.000, \\
0.001)\end{array}$ & 0.364 & 1.000 & 0.046 \\
\hline
\end{tabular}

$\mathrm{AAL}=$ Anatomical Automatic Labeling; $\mathrm{GM}=$ Gray Matter Volume; $\mathrm{WM}=$ White Matter Volume; VIF = Variance Inflation Factor

\section{Correlations between MMSE scores changes and brain volume changes}

There was a significant positive correlation between the GMV changes of left caudate nucleus and the MMSE scores changes in hemodialysis patients using Spearman's correlation analysis $\left(r_{s}=0.437, P=0.033\right)($ Fig. 3$)$.

\section{Discussion}

There were three vital findings in our study. First, compared with HCs,GMV in hemodialysis patients were diffusely decreased. Some regional GMV in hemodialysis patients at follow-up were also decreased compared to those patients at baseline. These findings indicate that hemodialysis patients suffered from brain atrophy over time. Second, the MMSE and MoCA scores of hemodialysis patients significantly lower than those in HCs. The MMSE scores in patients at follow-up were also significantly lower than those in patients at baseline. Furthermore, decreased GMV of left caudate nucleus showed significant positive correlation with reduced MMSE scores for the interval time between baseline and follow-up examinations, indicating that brain atrophy was associated with neurocognitive impairment. Third, long hemodialysis duration, increased serum urea, and increased parathyroid 
hormone were independent risk factors for regional GMV changes in hemodialysis patients by longitudinal analysis.

Our study found diffusely decreased GMV in hemodialysis patients compared with HCs, furthermore, decreased GMV in patients at follow-up compared with patients at baseline. These findings suggested that hemodialysis patients are more likely to suffer from GM atrophy over time. In our study, age, sex, and TIV of all subjects were taken as covariates to exclude the effects of these factors. The reasons for GM atrophy may be: First, hemodialysis patients are more likely to suffer from cerebrovascular diseases ${ }^{28}$ due to anemia ${ }^{29}$, oxidative stress ${ }^{30}$, and inflammation ${ }^{30}$. Cerebrovascular diseases have been reported as one of the factors to affect regional brain volumes. The occurrence of cerebrovascular diseases results in ischemia and hypoxia of regional brain tissues, which could further lead to brain tissue damage and showed the brain atrophy ${ }^{31}$. In our study, we found that the more increased parathyroid hormone, the more increased GM atrophy, which supported this reason. The increased parathyroid hormone can cause the secondary hyperparathyroidism and cause the metabolic disorder of calcium and phosphorus. These further increase the cerebrovascular diseases risk ${ }^{28}$. Second, renal failure leads to abnormal accumulation of organic toxic substances, such as urea, uric acid, guanidine compounds, and creatinine $^{32}$. Some studies have found that creatinine and guanidine compounds existed in the cerebrospinal fluid of uremia patients ${ }^{29,33}$. These neurotoxic substances may cause atrophy or death of glial cells and neurons, which further induce brain atrophy ${ }^{15}$. Our study also found that elevated serum urea can cause more decreased GM volumes, also supporting this finding. Third, a previous study had shown that iron deposition occurred in some brain regions of hemodialysis patients ${ }^{24}$, which can cause oxidative stress, further damage brain tissues and cause brain atrophy ${ }^{17}$. Fourth, we found that follow-up duration was a risk factor for reginal GMV decrease in hemodialysis patients. Patients were prone to cerebral ischemic vascular diseases due to the hemodynamic instability and decreased vascular autoregulation during the hemodialysis session ${ }^{34}$.

Our findings of decreased regional GMV in hemodialysis patients were partially consistent with some crosssectional studies ${ }^{15,16}$. However, there were still a few differences between our study and other studies ${ }^{15,16}$. In Zhang et al.'s study, they also found increased GMV of kidney failure patients mainly located in right extra-nuclear, right caudate, and right thalamus ${ }^{15}$. Gong et al. ${ }^{35}$ revealed increased regional GMV of hemodialysis patients in bilateral thalamus ${ }^{16}$, and bilateral caudate ${ }^{35}$. There are several possible explanations for these different findings: First, in the Gong et al.'s study ${ }^{35}$, all the hemodialysis patients were diagnosed with secondary hyperparathyroidism, However, the patients in our study were just hemodialysis patients. Although they had the increased parathyroid hormone levels, they cannot diagnose as the secondary hyperparathyroidism due to the clinical diagnostic criteria. Second, some studies have also suggested that different dialysis modalities have different effects on the incidence and degrees of cerebrovascular diseases, which may also cause different degrees of brain atrophy ${ }^{36}$. The study by Zhang et al. ${ }^{15}$ included both hemodialysis and peritoneal dialysis patients. However, the patients in our study were all hemodialysis patients. The comparison of effects of different dialysis modalities on the brain atrophy will need further investigation in the future.

We found that MMSE scores of patients at follow-up were significantly lower compared with patients at baseline, and the volumes changes of left caudate nucleus between two examinations were positively correlated with MMSE scores changes, indicating that brain atrophy was associated with the progressive neurocognitive impairment as the result of hemodialysis therapy. The caudate nucleus is an important part of frontostriatal brain circuit and it plays a role in cognitive function, especially executive function ${ }^{37}$. Some studies had found that caudate nucleus 
with discrete lesions caused executive function isolated deficits ${ }^{38}$. Some studies also demonstrated that the caudate nucleus was involved in complicated processes such as complex sentences and ambiguity resolution ${ }^{39}$. The MMSE test contained the high proportion of language test items, so it can correctly reflect the cognitive-related impairment caused by the decreased GMV of left caudate nucleus.

There were some limitations in our study. First, although our study explored longitudinal changes in brain volumes of hemodialysis patients, the HCs did not have follow-up examinations. The purpose of our study to observe the longitudinal changes of brain volumes in hemodialysis patients over time, so we mainly compared the brain volumes changes between patients at follow-up and baseline, between patients at baseline and HCs in our study. In order to avoid the effect of follow-up interval time on brain volumes, we also considered age, sex and TIV as covariates in the analysis. Second, the sample size of longitudinal study was relatively small, and a larger sample size is needed to explore the longitudinal brain volumes changes in hemodialysis patients. Third, our longitudinal study only relied on MMSE test, which may be not sensitive enough to mild cognitive impairment. MMSE also has some advantages including low false negative rate, short time consumption and simple operation in evaluation of moderate to severe cognitive impairment. It has been reported that the prevalence of mild to moderate cognitive impairment, severe impairment in patients were about $50 \%$ and $37 \%$. Besides, the MMSE had also been applied in some longitudinal studies to evaluate the cognitive function of some other diseases ${ }^{41}$. In the future, we will add other neuropsychological tests to evaluate cognitive function of patients.

\section{Conclusion}

Our cross-section and longitudinal study indicated that hemodialysis patients suffered from diffused GM atrophy over time, which correlated with cognitive impairment. Hemodialysis duration, altered levels of serum urea, and parathyroid hormone were independent risk factors for regional brain atrophy.

\section{Declarations}

\section{Acknowledgments}

We would like to express our gratitude to Professor E. Mark Haacke from Wayne State University who helped us with the spell, grammar, and the structures of manuscript.

\section{Declarations}

\section{Ethical Approval}

This study was approved by the Medical Research Ethics Committee of our hospital (2015N002KY).

\section{Consent to Participate}

All the subjects offered their written informed consent form.

\section{Consent to Publish}

All the authors agreed to publish this article.

\section{Authors Contributions}


Huiying Wang and Lixiang Huang substantial contributed to conception and design, acquisition of data, or analysis and interpretation of data; performing statistical analyses; drafting the article or revising it critically for important intellectual content. Gemuer Wu contributed to conception and design; evaluation and acquisition of data. Jinping Li, Lei Liu, Tong Zhang, Jinxia Zhu, Xianchang Zhang, and Wen Shen contributions to conception and design. Chao Chai and Shuang xia contributed to conception and design; drafting the article or revising it critically for important intellectual content; agreement to be accountable for all aspects of the work in ensuring that questions related to the accuracy or integrity of any part of the work are appropriately investigated and resolved.

\# Huiying Wang and Lixiang Huang participated to an equal extent in planning, analyzing, evaluating, and writing the paper.

* Chao Chai and Shuang Xia are co-corresponding authors.

\section{Funding}

This study has received funding by the Natural Scientific Foundation of China (grant number 81901728 to Chao Chaiðgrant number 81501457 to Shuang Xia『grant number 81873888 to Wen Shen), the National Key Technologies Research and Development Program of China (2019YFC0120901 to Shuang Xia), the Science and Technology talent cultivation Project of Tianjin Health Commission (grant number RC20185 to Chao Chai).

\section{Competing Interests}

The authors have no conflicts of interest to declare.

\section{Availability of data and materials}

A total of 97 hemodialysis patients were recruited from the Hemodialysis Center of our hospital from November 2015 to July 2019. Seventy-six age-, sex-, and education-matched HCs were recruited from local communities. The blood biochemical examinations were performed using a Vitros 350 Automatic Biochemical Analyzer (Johnson \& Johnson, Warren, NJ) within a week before MR scanning. All MRI data were acquired on a $3 \mathrm{~T}$ MR imager (MAGNETOM Trio a Tim System, Siemens Healthcare) using an 8-channel phased-array head coil.

\section{References}

1. Chronic kidney disease. (2015). in adults: assessment and management. London: National Institute for Health and Care Excellence (UK).

2. Ghoshal, S., \& Freedman, B. I. (2019). Mechanisms of Stroke in Patients with Chronic Kidney Disease. AM J NEPHROL, 50(4), 229-239.

3. Findlay, M. D., Dawson, J., Dickie, D. A., et al. (2019). Investigating the Relationship between Cerebral Blood Flow and Cognitive Function in Hemodialysis Patients. J AM SOC NEPHROL, 30(1), 147-158.

4. Freire, D. M. C., Diogenes, D. S. B., Costa, B. G., et al. (2020). Cognitive impairment, endothelial biomarkers and mortality in maintenance haemodialysis patients: a prospective cohort study. Nephrol Dial Transplant, 35(10), 1779-1785.

5. Alqahtani, F., Berzingi, C. O., Aljohani, S., et al. Temporal Trends in the Outcomes of Dialysis Patients Admitted With Acute Ischemic Stroke. J AM HEART ASSOC 2018;7(12). 
6. McQuillan, R., \& Jassal, S. V. (2010). Neuropsychiatric complications of chronic kidney disease. NAT REV NEPHROL, 6(8), 471-479.

7. Jin, M., Wang, L., Wang, H., et al. (2020). Altered resting-state functional networks in patients with hemodialysis: a graph-theoretical based study. BRAIN IMAGING BEHAV.

8. Chou, M. C., Ko, C. H., Chang, J. M., \& Hsieh, T. J. (2019). Disruptions of brain structural network in end-stage renal disease patients with long-term hemodialysis and normal-appearing brain tissues. J Neuroradiol, 46(4), 256-262.

9. Chai, C., Zhang, M., Wang, H., et al. (2020). Increased cerebral blood flow is correlated with neurocognitive impairment in long-term hemodialysis patients: an arterial spin labeling MRI study. BRAIN IMAGING BEHAV.

10. Ma, X., Zhang, Y., Ma, S., et al. (2018). Association between abnormal thalamic metabolites and sleep disturbance in patients with end-stage renal disease. METAB BRAIN DIS, 33(5), 1641-1648.

11. Chai, C., Wang, Z., Fan, L., et al. (2016). Increased Number and Distribution of Cerebral Microbleeds Is a Risk Factor for Cognitive Dysfunction in Hemodialysis Patients: A Longitudinal Study. Medicine, 95(12), e2974.

12. Wu, B., Li, X., Zhang, M., et al. (2020). Disrupted brain functional networks in patients with end-stage renal disease undergoing hemodialysis. J NEUROSCI RES, 98(12), 2566-2578.

13. Prohovnik, I., Post, J., Uribarri, J., Lee, H., Sandu, O., \& Langhoff, E. (2007). Cerebrovascular effects of hemodialysis in chronic kidney disease. J Cereb Blood Flow Metab, 27(11), 1861-1869.

14. Drew, D. A., Bhadelia, R., Tighiouart, H., et al. (2013). Anatomic brain disease in hemodialysis patients: a crosssectional study. AM J KIDNEY DIS, 61(2), 271-278.

15. Zhang, L. J., Wen, J., Ni, L., et al. (2013). Predominant gray matter volume loss in patients with end-stage renal disease: a voxel-based morphometry study. METAB BRAIN DIS, 28(4), 647-654.

16. Jin, M., Wang, L., Wang, H., et al. (2020). Structural and Functional Alterations in Hemodialysis Patients: A Voxel-Based Morphometry and Functional Connectivity Study. FRONT HUM NEUROSCI, 14, 80.

17. Chai, C., Zhang, M., Long, M., et al. (2015). Increased brain iron deposition is a risk factor for brain atrophy in patients with haemodialysis: a combined study of quantitative susceptibility mapping and whole brain volume analysis. METAB BRAIN DIS, 30(4), 1009-1016.

18. Tsuruya, K., Yoshida, H., Kuroki, Y., et al. (2015). Brain atrophy in peritoneal dialysis and CKD stages 3-5: a cross-sectional and longitudinal study. AM J KIDNEY DIS, 65(2), 312-321.

19. Lu, R., Kiernan, M. C., Murray, A., Rosner, M. H., \& Ronco, C. (2015). Kidney-brain crosstalk in the acute and chronic setting. NAT REV NEPHROL, 11(12), 707-719.

20. Farokhian, F., Beheshti, I., Sone, D., \& Matsuda, H. (2017). Comparing CAT12 and VBM8 for Detecting Brain Morphological Abnormalities in Temporal Lobe Epilepsy. FRONT NEUROL, 8, 428.

21. Kubera, K. M., Schmitgen, M. M., Nagel, S., et al. (2019). A search for cortical correlates of trait impulsivity in Parkinson s disease. BEHAV BRAIN RES, 369, 111911.

22. Seiger, R., Ganger, S., Kranz, G. S., Hahn, A., \& Lanzenberger, R. (2018). Cortical Thickness Estimations of FreeSurfer and the CAT12 Toolbox in Patients with Alzheimer's Disease and Healthy Controls. $J$ NEUROIMAGING, 28(5), 515-523.

23. Schmitgen, M. M., Depping, M. S., Bach, C., et al. (2019). Aberrant cortical neurodevelopment in major depressive disorder. J Affect Disord, 243, 340-347.

24. Chai, C., Wang, H., Liu, S., et al. (2019). Increased iron deposition of deep cerebral gray matter structures in hemodialysis patients: A longitudinal study using quantitative susceptibility mapping. J MAGN RESON 
IMAGING, 49(3), 786-799.

25. Jiang, W., Hu, C. Y., Li, F. L., Hua, X. G., Huang, K., \& Zhang, X. J. (2020). Elevated parathyroid hormone levels and cognitive function: A systematic review. Arch Gerontol Geriatr, 87, 103985.

26. Chai, C., Wang, H., Chu, Z., et al. (2020). Reduced regional cerebral venous oxygen saturation is a risk factor for the cognitive impairment in hemodialysis patients: a quantitative susceptibility mapping study. BRAIN IMAGING BEHAV, 14(5), 1339-1349.

27. Lin, C., Lin, H., Wang, S., \& Fuh, J. (2020). Association between regional brain volume and masticatory performance differed in cognitively impaired and non-impaired older people. EXP GERONTOL, 137, 110942.

28. Kelly, D., \& Rothwell, P. M. (2020). Disentangling the multiple links between renal dysfunction and cerebrovascular disease. J Neurol Neurosurg Psychiatry, 91(1), 88-97.

29. Brancaccio, D., Cozzolino, M., \& Gallieni, M. (2004). Hyperparathyroidism and anemia in uremic subjects: a combined therapeutic approach. J AM SOC NEPHROL, 15(Suppl 1), S21-S24.

30. Rapa, S. F., Di lorio, B. R., Campiglia, P., Heidland, A., \& Marzocco, S. Inflammation and Oxidative Stress in Chronic Kidney Disease-Potential Therapeutic Role of Minerals, Vitamins and Plant-Derived Metabolites. INT J MOL SCI 2019;21(1).

31. Pedraza, M. I., de Lera, M., Bos, D., et al. Brain Atrophy and the Risk of Futile Endovascular Reperfusion in Acute Ischemic Stroke. STROKE 2020;51(5):1514-1521.

32. Marini, S., Georgakis, M. K., Chung, J., et al. (2020). Genetic overlap and causal inferences between kidney function and cerebrovascular disease. NEUROLOGY, 94(24), e2581-e2591.

33. De Deyn, P. P., Vanholder, R., Eloot, S., \& Glorieux, G. (2009). Guanidino compounds as uremic (neuro)toxins. Semin Dial, 22(4), 340-345.

34. Wolfgram, D. F. (2019). Intradialytic Cerebral Hypoperfusion as Mechanism for Cognitive Impairment in Patients on Hemodialysis. J AM SOC NEPHROL, 30(11), 2052-2058.

35. Gong, X., Zou, L., Wu, H., et al. (2020). Altered brain structural and cognitive impairment in end-stage renal disease patients with secondary hyperparathyroidism. ACTA RADIOL, 61(6), 796-803.

36. Drew, D. A., \& Sarnak, M. J. (2014). Ischemic and hemorrhagic stroke: high incidence in hemodialysis and peritoneal dialysis patients. AM J KIDNEY DIS, 63(4), 547-548.

37. Jeon, H. A., Anwander, A., \& Friederici, A. D. (2014). Functional network mirrored in the prefrontal cortex, caudate nucleus, and thalamus: high-resolution functional imaging and structural connectivity. $\mathrm{J} \mathrm{NEUROSCl}$, 34(28), 9202-9212.

38. Postuma, R. B., \& Dagher, A. (2006). Basal ganglia functional connectivity based on a meta-analysis of 126 positron emission tomography and functional magnetic resonance imaging publications. CEREB CORTEX, 16(10), 1508-1521.

39. Mestres-Misse, A., Turner, R., \& Friederici, A. D. (2012). An anterior-posterior gradient of cognitive control within the dorsomedial striatum. NEUROIMAGE, 62(1), 41-47.

40. Murray, A. M., Tupper, D. E., Knopman, D. S., et al. (2006). Cognitive impairment in hemodialysis patients is common. NEUROLOGY, 67(2), 216-223.

41. Fruehwirt, W., Dorffner, G., Roberts, S., et al. (2019). Associations of event-related brain potentials and Alzheimer's disease severity: A longitudinal study. Prog Neuropsychopharmacol Biol Psychiatry, 92, 31-38.

\section{Figures}




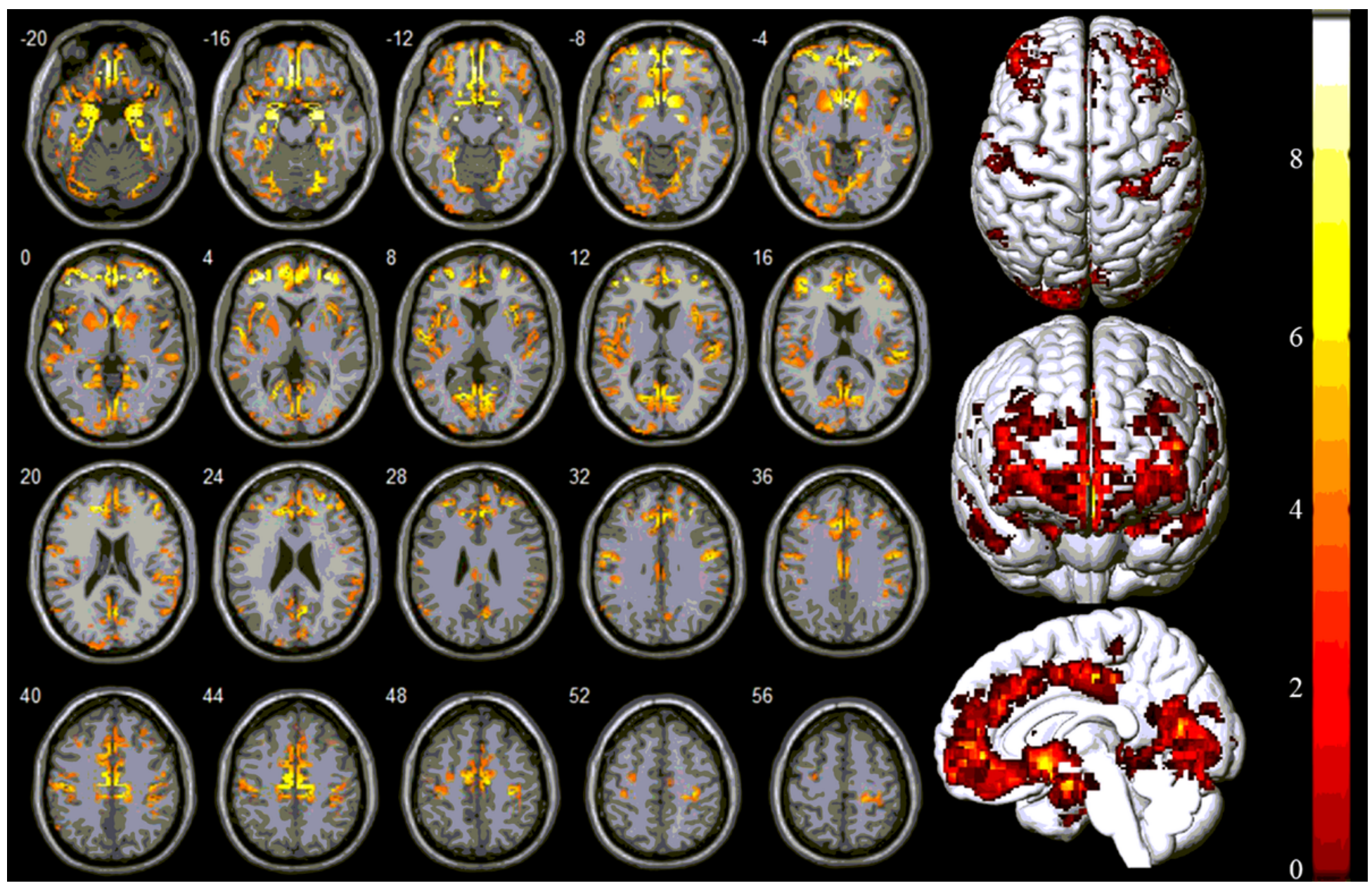

Figure 1

Brain regions with significantly decreased gray matter volume between hemodialysis patients and HCs Compared to HCs, hemodialysis patients showed significantly decreased GMV in bilateral frontal lobes, temporal lobes, occipital lobe, parietal lobe, limbic system, caudate, putamen, and globus pallidus (FDR corrected with age, sex, and TIV as covariates; voxel-wise threshold of $\mathrm{P}<0.001$ ) 


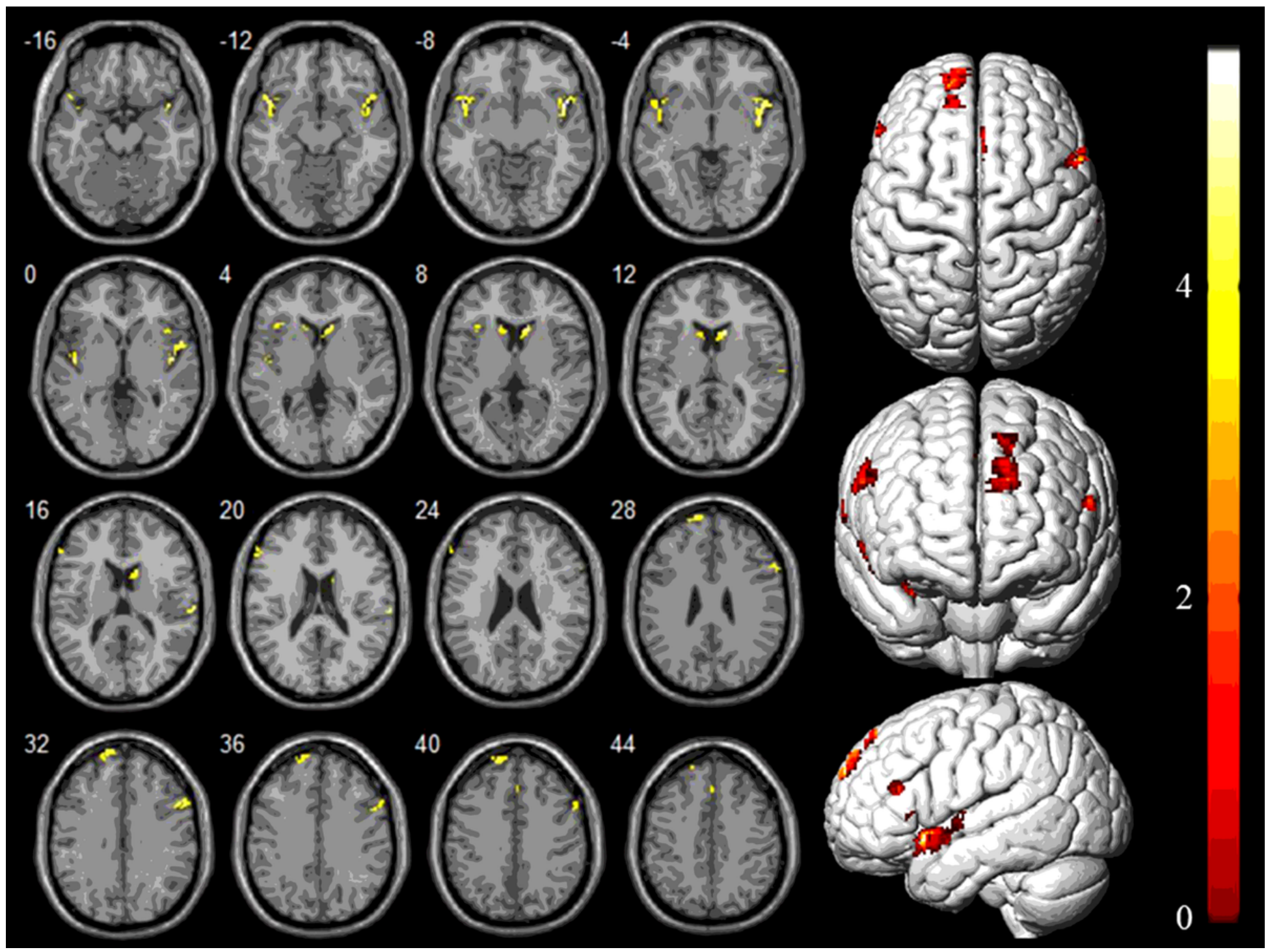

Figure 2

Brain regions with significantly decreased gray matter volume between baseline and follow-up patients Compared to patients at baseline, patients at follow-up showed significantly decreased GMV in the bilateral frontal lobes, temporal lobes, insula, Rolandic operculum, caudate, right supplementary motor area, right precentral gyrus, right supramarginal gyrus, and right cingulate gyrus (with age and TIV as covariates, voxel-wise threshold of $P<0.05$, FDR corrected) 


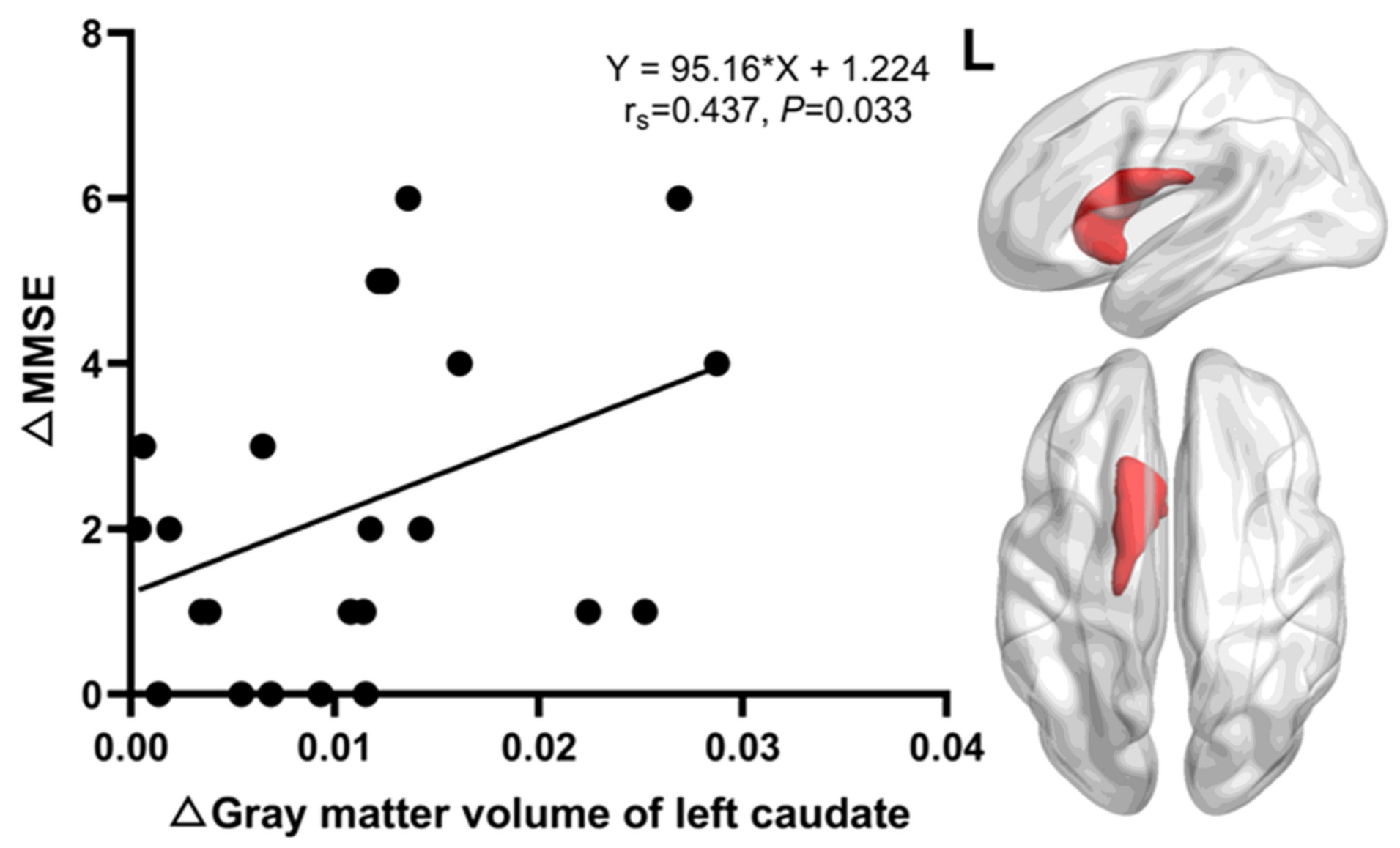

Figure 3

Correlation between changes of left caudate volume and changes of MMSE score The changes in the GM volume of left caudate nucleus between baseline and follow-up examinations were positively correlated with the changes of MMSE score ( $r s=0.437, \mathrm{P}=0.033)$

\section{Supplementary Files}

This is a list of supplementary files associated with this preprint. Click to download.

- supplementalMaterials.docx 